\title{
The Validity of Registered Synchronous Peritoneal Metastases from Colorectal Cancer in the Danish Medical Registries
}

This article was published in the following Dove Press journal: Clinical Epidemiology

\author{
Sissel Ravn (D) \\ Christian F Christiansen (iD ${ }^{2}$ \\ Rikke H Hagemann- \\ Madsen ${ }^{3}$ \\ Victor J Verwaal' \\ Lene $\mathrm{H}$ Iversen $^{1,4}$ \\ 'Department of Surgery, Aarhus \\ University Hospital, Aarhus, Denmark; \\ ${ }^{2}$ Department of Clinical Epidemiology, \\ Aarhus University Hospital, Aarhus, \\ Denmark; ${ }^{3}$ Department of Pathology, \\ Lillebaelt Hospital, Vejle, Denmark; \\ ${ }^{4}$ Danish Colorectal Cancer Group \\ (DCCG), Copenhagen, Denmark
}

Introduction: Treatment options for peritoneal metastases (PM) from colorectal cancer (CRC) have increased, their efficiency should be monitored. For this purpose, register-based data on PM can be used, if valid.

Purpose: We aimed to evaluate the completeness and positive predictive value (PPV) of synchronous peritoneal metastases (S-PM) registered among CRC patients in the Danish National Patient Register (DNPR) and/or the Danish National Pathology Register (the DNPatR) using the Danish Colorectal Cancer Group database (DCCG) as a reference.

Patients and Methods: We identified Danish patients with newly diagnosed primary CRC in the DCCG during 2014-2015. S-PM were routinely registered in the DCCG. We excluded patients with non-CRC cancers and identified S-PM using all three registries. We estimated the completeness and the PPV of registered S-PM in the DNPR, the DNPatR and the DNPR and/or the DNPatR (DNPR/DNPatR) in combination using the DCCG as the reference. We stratified by age, gender, WHO performance status, tumour location and distant metastases to liver and/or lungs.

Results: We identified 9142 patients with CRC in DCCG. In DCCG, 366 patients were registered with S-PM, among whom 213 in DCCG only, whereas 153 in DCCG and in at least one of DNPR and/or DNPatR. In DNPR/DNPatR, S-PM was registered with a completeness of $42 \%$ [95\% CI: 37-47] and a PPV of $60 \%$ [95\% CI: 54-66]. In the DNPR only, the completeness was 32\% [95\% CI: 27-37] and the PPV 57\% [95\% CI: 5064]. The completeness in the DNPatR was 19\% [95\% CI: 15-23] and the PPV was $76 \%$ [95\% CI: 68-85]. In the DNPR/DNPatR patients aged <60 years (57\% [95\% CI: 46-69]), patients with WHO performance status 0 (46\% [95\% CI: 37-54]) and patients with no distant metastases (58\% [95\% CI: 50-65]) were registered with a higher completeness.

Conclusion: Our algorithm demonstrates that the DNPR/DNPatR captures less than half of CRC patients with S-PM. Potential candidates for curative treatment options are registered with a higher completeness. Clinicians should be encouraged to register the presence of S-PM to increase the validity of register-based S-PM data.

Keywords: validity, synchronous peritoneal metastases, registries, colorectal cancer, epidemiology, completeness

\section{Introduction}

Colorectal cancer (CRC) is the third most common cancer worldwide and in Denmark. ${ }^{1}$ Approximately $18-19 \%$ of CRC patients present with metastatic disease. ${ }^{2,3}$ Treatment of metastatic CRC in particular has improved considerably during the past decade, primarily due to a multidisciplinary approach offering metastasis-directed treatment. Such treatment options include, among others, surgical resection, ablative procedures
Correspondence: Sissel Ravn Department of Surgery, Aarhus University Hospital, Aarhus N DK-8200, Denmark

Tel +4551627366

Email sissel.ravn@rm.dk 
(radiofrequency ablation (RFA) or microwave therapy), stereotactic radiotherapy, and cytoreductive surgery (CRS) and hyperthermic intraperitoneal chemotherapy (HIPEC). ${ }^{4,5}$ Treatment strategies for colorectal peritoneal metastases (PM) have changed from palliative treatment to intended curative treatment with CRS and HIPEC. ${ }^{6,7}$ PM diagnosed concurrently with the primary CRC is referred to as synchronous peritoneal metastases (S-PM), while recurrence in the peritoneum is called metachronous peritoneal metastases. There is no well-defined distinction between synchronous and metachronous PM, however, an interval of 6 (or less) months after diagnosis of the primary $\mathrm{CRC}$ is often used to define synchronous PM (S-PM). ${ }^{8,9}$

The diagnosis of PM is challenging and not necessarily based on a histopathological verification. The sensitivity of a PM diagnosis is based on preoperative radiological assessment such as computer tomography (CT) scans, and has been reported with a great variance from $11 \%$ to $96 \% .{ }^{10}$ Currently, no radiological imaging is superior to an intra-operative assessment of the peritoneal cavity. ${ }^{11}$

Registries contain large comprehensive data, and the registration of S-PM enables clinicians and researchers to monitor prevalence and incidence along with treatment methods, mortality rates and the prognosis. However, this relies on the validity of S-PM data. ${ }^{12}$ To our knowledge, the completeness of registered metastasis from CRC is poorly investigated. An algorithm to identify CRC recurrence in registries has been developed by Lash et al $;{ }^{13}$ however, the algorithm is not specific for anatomic site of recurrence, especially not in the peritoneum. When investigating the validity of register-based data on metastasis, the value of medical charts as the golden standard has been questioned because metastasis not necessarily leads to medical attention, diagnostics and registration. ${ }^{14,15}$ No algorithm to identify S-PM from CRC has been developed.

We aimed to evaluate the completeness and positive predictive value (PPV) of the registration of S-PM in the Danish medical registries.

\section{Methods}

\section{Study Design and Setting}

The study was designed as a nationwide population-based prevalence study using data from the Danish National Colorectal Cancer Group (DCCG) database, the Danish Civil Registration System (DCRS), the Danish National Patient Registry (DNPR) and The Danish National Pathology Registry (DNPatR). The study is reported according to the "Strengthening the Reporting of Observational Studies in Epidemiology (STROBE)" guidelines. ${ }^{16}$ We included all Danish patients registered in the DCCG database with primary CRC diagnosed in the period January 1st 2014 to December 31st 2015. The population of Denmark includes approximately 5.8 million inhabitants. All Danish citizens have access to a tax-supported primary and secondary health-care system. ${ }^{17}$

\section{Study Population}

The date of CRC diagnosis as registered in the DCCG database was defined as the index date. Patients with another primary, non-CRC diagnosis registered in the DNPR within a period of 5 years prior to and 180 days after index date were excluded to ensure that PM originated from CRC (Figure 1). However, patients remained in the study if S-PM was identified in the DCCG database or histopathological verified to originate from CRC.

\section{Data Sources}

\section{The Danish Colorectal Cancer Group}

Since 2001, Danish patients with first-time, primary CRC managed at a surgical department have been registered in the DCCG database. The date of CRC is registered as the date of biopsy verification. If CRC is not histologically verified, the date of CRC diagnosis is registered as the date of surgery or the date when the patient is informed of the cancer. The completeness of CRC patients in the DCCG is $>95 \% .{ }^{18}$ The DCCG database contains patient-related characteristics and information on diagnostic, surgical, pathological and few oncological procedures. All information is registered within 30 days from the CRC diagnosis. Recurrence of the CRC is not registered in the database. The DCCG database underwent a major revision in 2009 including more pathology and again in 2014, implicating an implementation of more detailed registration of several variables, including the registration of S-PM.

\section{The Danish Civil Registration System}

All Danish residents are registered in the DCRS, and assigned with a unique 10-digit civil person registration (CPR) number, entailing unambiguous individual-level record linkage to other Danish registers. The DCRS is updated with information on migration and vital status on a daily basis, allowing complete long-term follow-up. ${ }^{19}$

\section{The Danish National Patient Registry}

It is mandatory for all hospitals in Denmark to report information on all outpatient and inpatient hospital 


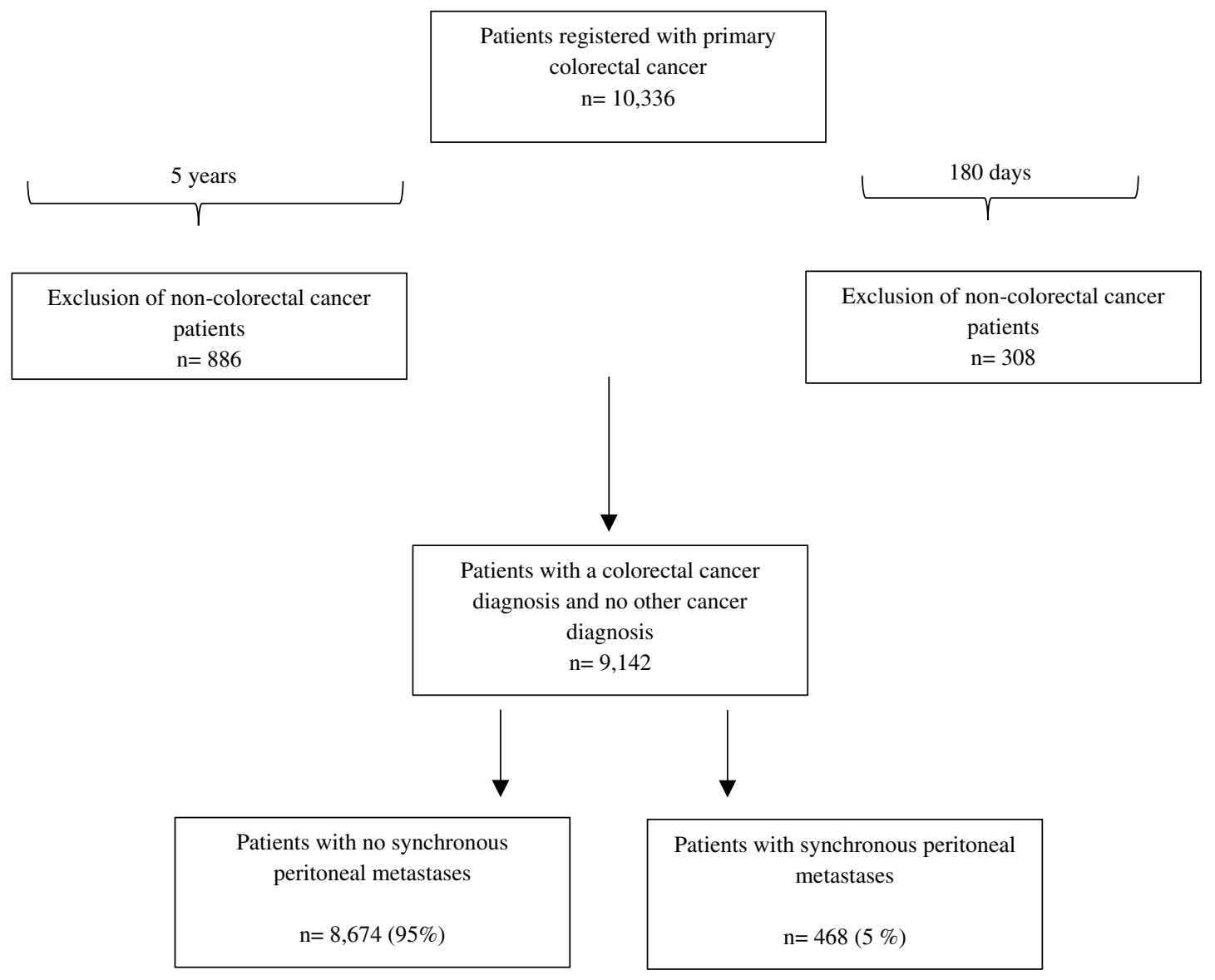

Figure I Flowchart of Danish colorectal cancer patients diagnosed with synchronous peritoneal metastases (S-PM) between 2014 and 2015.

contacts to the DNPR. For each hospitalization, DNPR record dates of admission and discharge, one primary and potentially several secondary diagnoses using the Danish version of the International Classification 8th revision (ICD-8) from 1977 to 1993 and ICD-10 thereafter. ${ }^{12}$

\section{The Danish National Pathology Registry}

The DNPatR was established in 1997 and all pathological examinations performed in Denmark are registered according to national guidelines, including a topography- and morphology-code as a minimum. Each specimen is linked to the patient's CPR and the specific Danish Systematized Nomenclature of Medicine (SNOMED) codes. ${ }^{20}$

\section{The Reference Database}

We choose to use the DCCG database as the reference standard because we expected that these data would be of the highest completeness since the presence or absence of
PM is mandatorily registered by dedicated CRC-surgeons since 2014.

In general, the reference standard often used to validate register-based data is medical journals. However, the quality of medical journals as "golden standard" in the validation of registered metastases has been questioned, mainly because medical charts itself may be incomplete with respect to recording the presence and/or exact numbers and onset of metastases. ${ }^{15}$ Further, some metastases may be asymptomatic and may not come to medical attention until routine checkups, and it is possible that recording of metastases in the medical charts is not prioritized for patients with a limited life expectancy. ${ }^{15}$

The registration of $\mathrm{PM}$ is even more difficult because the diagnosis of PM is challenging in its nature. For example, there is a large variability in the sensitivity of the radiological imaging ranging from $\mathrm{CT}$ scans, PET/CT and MRI and the variability is dependent on the anatomical site and size of the peritoneal metastases. ${ }^{10}$ 


\section{Clinical Variables}

All clinical- and patient-related variables were collected from the DCCG database. The Charlson Comorbidity Index (CCI) was categorized into three levels of comorbidity ( 0 : no comorbidity, $1-2$ : mild to moderate comorbidity, and $>2$ : severe comorbidity). ${ }^{21}$

\section{Algorithm for Identification of Synchronous Peritoneal Metastases}

The algorithm applied to identify S-PM patients included the identification of a PM diagnosis registered in at least one of the three registries (the DCCG database, the DNPR and the DNPatR) within 180 days after the date of CRC diagnosis.

For specific ICD-10 codes in the DNPR and specific SNOMED codes in the DNPatR please see the Appendix.

\section{Statistical Analysis}

To validate the registration of the ICD-10 codes in the DNRP and the SNOMED codes in the DNPatR we used registrations in the DCCG as reference.

The sensitivity formula was used as a measure of completeness, and estimated for each registry (DNPR and DNPatR) and for the registries in combination (DNPR/DNPatR).

The completeness was estimated by dividing the number of patients with a PM diagnosis in both the respective registry (DNPR, DNPatR or a combination (DNPR/DNPatR)) and the DCCG (numerator) by the number of all patients registered with PM in the DCCG (denominator).

The PPV was estimated for each registry (DNPR and DNPatR) and for the registries in combination (DNPR/ DNPatR). The PPV was defined as the number of patients registered with a $\mathrm{PM}$ diagnosis in the respective registry (DNPR, DNPatR or a (DNPR/DNPatR)) and the DCCG (numerator), divided by the number of all patients with a PM diagnosis registered in the respective registry (DNPR, DNPatR or a combination).

We used 180 days after the index date to distinguish between synchronous and metachronous PM. The median days from CRC diagnosis until diagnosis of S-PM in one or more of the registries were presented with the interquartile range. The date of S-PM diagnosis in the DCCG was equal to the date of CRC cancer because this was registered concurrently. If patients were registered in both the DCCG and the DNPR/DNPatR, the date of S-PM in the DNPR/DNPatR was used, which ever came first.
To evaluate if the coding quality in the DNPR/DNPatR differed within clinical relevant subgroups, we stratified by age groups $(\leq 60,60-69,70-74,75-80$ and $\geq 80$ years), sex, WHO performance status, tumour location and distant metastases to the liver and/or the lungs (yes/no).

Patient characteristics are presented by patients registered in (1) Only the DCCG, (2) the DCCG and the DNPR/ DNPatR, (3) only the DNPR/DNPatR and (4) the total number of patients registered with S-PM. Categorical variables are presented as numbers with percentages, whereas continuous variables are presented as the median with range. Statistical analyses are performed with STATA ${ }^{\circledR}$ software (version 15.1, STATA, College Station, TX, USA).

\section{Ethics}

The study was registered by the Danish Data Protection Agency through the Central Region of Denmark (record number 1-16-02-441-16). Ethical approval is not necessary for non-interventional register-based studies in Denmark.

\section{Results}

During 2014-2015, 10,336 patients were diagnosed with primary CRC and identified in the DCCG database among whom 1194 patients were excluded due to a diagnosis of non-colorectal cancer within 5 years prior to or 180 days after the index date (Figure 1).

By the use of our algorithm, 366/9142 (4\%) patients were registered with S-PM in the DCCG, among whom 153 patients were registered in all registries (DCCG, DNPR and DNPatR). Furthermore, we identified 102/ $9142(1 \%)$ patients with a S-PM diagnosis registered in only the DNPR/DNPatR (Figure 2).

\section{Patient Characteristics}

We compared patients registered with S-PM in (1) only the DCCG, (2) the DCCG and the DNPR/DNPatR, and (3) only the DNPR/DNPatR (Table 1). Regarding the patients who were only registered in the DNPR/ DNPatR: A marginal larger proportion was $<60$ years, while a larger proportion was males, presented with WHO performance status 0 , had a rectal tumour and no distant metastases (apart from PM). Information on (y)pT-and (y)pN-categories were missing for $67 \%$ and $68 \%$ of all registered S-PM patients, presumably because few S-PM patients underwent initial surgery of the primary CRC tumour. However, this information was only missing for $43 \%$ and $44 \%$ of the patients 


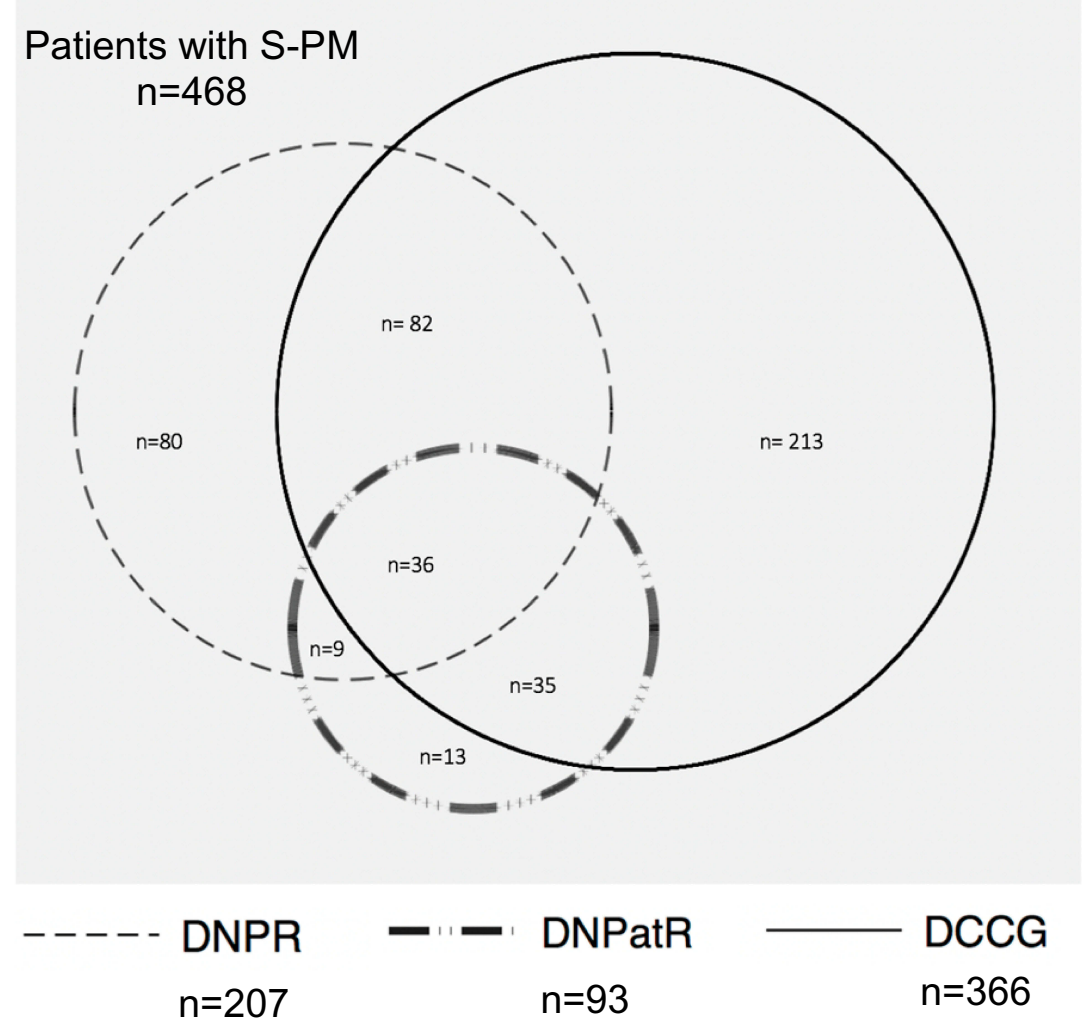

Figure 2 Number of patients diagnosed with synchronous peritoneal metastases (S-PM) in one or more of the 3 registries: The Danish Colorectal Cancer Group (DCCG) database, The Danish National Patient Registry (DNPR) and the Danish National Pathology Registry (DNPatR) during 20I4-2015.

registered only in the DNPR/DNPatR. Among the patients only registered in the DNPR/DNPatR, $42 \%$ had a (y)pT4 tumour and 33\% presented with a (y)pN2category.

\section{Registration of Synchronous Peritoneal Metastasis in the Different Registries}

Of the 468 patients registered with S-PM, 78\% were identified in the DCCG, whereas $45 \%$ were registered only in the DCCG, 33\% were registered in both the DCCG and the DNPR/DNPatR, while 22\% were registered only in the DNPR/DNPatR (Table 2 and Figure 2).

\section{Completeness and Positive Predictive Value}

Using a combination of both the DNPR/DNPatR, we found a completeness of $42 \%$ [95\% CI: 37-47] and a PPV of $60 \%$ [95\% CI: 54-66] (Table 3).

By the use of the DNPR only, the completeness was $32 \%$ [95\% CI: $27-37$ ] and the PPV 57\% [95\% CI: 50-64] (Table 3). When using only the DNPatR, the completeness was $19 \%$ [95\% CI: $15-23]$ and the PPV was $76 \%[95 \%$ CI: 68-85] (Table 3).

\section{Stratified Analysis}

According to our algorithm, the stratified analysis showed that some subgroups were registered with a higher completeness compared to the overall completeness (42\% [95\% CI: 37-47]). These groups were patients with age $<60$ years $(57 \%$ [95\% CI: 46-69]), WHO performance status 0 (46\% [95\% CI: 37-54]), and no distant metastases (58\% [95\% CI: 50-65]) (apart from PM) (Table 4). Contrary, some subgroups were registered with a lower completeness: patients aged $>80$ years $(32 \%$ [95\% CI: 22-42]), patients with a WHO performance status 2 (32\% [95\% CI: 20-44]), patients with rectal tumours (33\% [95\% CI: 19-48]), and patients with distant metastases (29\% [95\% CI: 22-35]) (apart from PM) Table 4).

\section{Days from Colorectal Cancer to}

\section{Synchronous Peritoneal Metastases}

Median days from the diagnosis of CRC to the diagnosis of S-PM varied according to each register. When 
Table I Baseline Characteristics

\begin{tabular}{|c|c|c|c|c|}
\hline Patients Characteristics & $\begin{array}{c}\text { Patients } \\
\text { Registered Only } \\
\text { in the DCCG }\end{array}$ & $\begin{array}{c}\text { Patients Registered in the } \\
\text { DCCG and DNPR and/or } \\
\text { DNPatR }\end{array}$ & $\begin{array}{c}\text { Patients Registered } \\
\text { Only in the DNPR/ } \\
\text { DNPatR }\end{array}$ & $\begin{array}{c}\text { Total Number of } \\
\text { Patients Registered } \\
\text { with S-PM }\end{array}$ \\
\hline & $n=213$ & $n=153$ & $n=102$ & $n=468$ \\
\hline Median age (years, range) & $72(18-94)$ & $69(33-97)$ & 72.5 (48-9I) & 71 (18-97) \\
\hline $\begin{array}{l}\text { Age Groups } \\
\begin{array}{l}<60 \\
60-69 \\
70-74 \\
75-80 \\
>80\end{array}\end{array}$ & $\begin{array}{l}29(14) \\
58(27) \\
37(17) \\
36(17) \\
53(25)\end{array}$ & $\begin{array}{l}39(25) \\
39(25) \\
27(18) \\
23(15) \\
25(16)\end{array}$ & $\begin{array}{l}21(21) \\
21(21) \\
17(17) \\
26(25) \\
17(17)\end{array}$ & $\begin{array}{l}89(19) \\
118(25) \\
81(17) \\
85(18) \\
35(20)\end{array}$ \\
\hline $\begin{array}{l}\text { Sex } \\
\text { Female } \\
\text { Male }\end{array}$ & $\begin{array}{l}116(54) \\
97(46)\end{array}$ & $\begin{array}{l}74(48) \\
79(52)\end{array}$ & $\begin{array}{l}41(40) \\
61(60)\end{array}$ & $\begin{array}{l}231(49) \\
237(5 I)\end{array}$ \\
\hline $\begin{array}{l}\text { Comorbidity, Charlson Score } \\
\begin{array}{l}0 \\
\mathrm{I}-2 \\
>2\end{array}\end{array}$ & $\begin{array}{l}127(60) \\
56(26) \\
30(14)\end{array}$ & $\begin{array}{l}89(58) \\
39(25) \\
25(16)\end{array}$ & $\begin{array}{l}63(62) \\
25(24) \\
14(14)\end{array}$ & $\begin{array}{l}279(60) \\
120(26) \\
69(14)\end{array}$ \\
\hline $\begin{array}{l}\text { WHO Performance Status } \\
\text { Status } 0 \\
\text { Status I } \\
\text { Status } 2 \\
\text { Status >2 } \\
\text { Missing }\end{array}$ & $\begin{array}{l}70(33) \\
61(29) \\
38(18) \\
22(10) \\
22(10)\end{array}$ & $\begin{array}{l}59(39) \\
50(33) \\
18(12) \\
13(8) \\
13(8)\end{array}$ & $\begin{array}{l}48(47) \\
26(25) \\
14(14) \\
6(6) \\
8(8)\end{array}$ & $\begin{array}{l}177(38) \\
137(29) \\
70(15) \\
41(9) \\
43(9)\end{array}$ \\
\hline $\begin{array}{l}\text { Tumour Location } \\
\text { Colon } \\
\text { Rectum }\end{array}$ & $\begin{array}{l}185(87) \\
28(13)\end{array}$ & $\begin{array}{c}139(91) \\
14(9)\end{array}$ & $\begin{array}{l}85(83) \\
17(17)\end{array}$ & $\begin{array}{c}409(87) \\
59(13)\end{array}$ \\
\hline $\begin{array}{l}\text { Pathological }(\mathrm{y}) \mathrm{PT}^{\mathrm{a}} \text {-Category } \\
\text { T0 + TI } \\
\text { T2 } \\
\text { T3 } \\
\text { T4 } \\
\text { Tx } \\
\text { Missing }\end{array}$ & $\begin{array}{c}2(1) \\
1(0) \\
10(5) \\
39(18) \\
0(0) \\
161(76)\end{array}$ & $\begin{array}{c}0(0) \\
0(0) \\
4(3) \\
4 I(27) \\
0(0) \\
108(71)\end{array}$ & $\begin{array}{c}I(1) \\
I(1) \\
12(12) \\
43(42) \\
I(1) \\
44(43)\end{array}$ & $\begin{array}{c}3(1) \\
2(0) \\
26(6) \\
123(26) \\
1(0) \\
313(67)\end{array}$ \\
\hline $\begin{array}{l}\text { Pathological (y)p } \mathrm{N}^{\mathrm{b}} \text {-Category } \\
\text { No } \\
\mathrm{NI} \\
\mathrm{N} 2 \\
\mathrm{Nx}\end{array}$ & $\begin{array}{c}4(2) \\
21(10) \\
25(12) \\
163(77)\end{array}$ & $\begin{array}{c}6(4) \\
14(9) \\
25(16) \\
108(71)\end{array}$ & $\begin{array}{l}10(10) \\
15(15) \\
33(33) \\
44(44)\end{array}$ & $\begin{array}{c}20(4) \\
50(11) \\
83(18) \\
315(68)\end{array}$ \\
\hline $\begin{array}{l}\text { Distant Metastases to Either } L \\
\text { Liver } \\
\text { Yes } \\
\text { No }\end{array}$ & $\begin{array}{c}143(67) \\
70(33)\end{array}$ & $\begin{array}{l}57(38) \\
96(63)\end{array}$ & $\begin{array}{l}4 I(40) \\
6 I(60)\end{array}$ & $\begin{array}{l}24 \mid(52) \\
227(48)\end{array}$ \\
\hline
\end{tabular}

Notes: Patients registered with Synchronous peritoneal metastases (S-PM) from colorectal cancer. Data are presented as number of patients (\%) with registration in I) Only the Danish Colorectal Cancer Group (DCCG), 2) Both the DCCG and the Danish National Patient Registry (DNPR) and/or the Danish National Pathology Registry

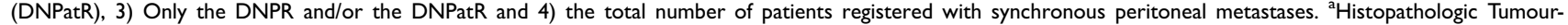
category. $T$ indicates the size and extension of the tumour; $P$ indicates that the category is given by a histopathologic examination and $y$ indicates that the category is assessed after chemotherapy and/or radiation therapy. ${ }^{b}$ Histopathologic Lymph node-category. $\mathrm{N}$ indicates the degree of spread to regional lymph nodes; $\mathrm{p}$ indicates that the category is given by a histopathologic examination and $y$ indicates that the category is assessed after chemotherapy and/or radiation therapy. 
Table 2 Number of Patients Diagnosed in One or More of the 3 Registries

\begin{tabular}{|c|c|c|c|c|}
\hline $\begin{array}{l}\text { Only } \\
\text { DCCG }\end{array}$ & $\begin{array}{l}\text { DCCG and } \\
\text { DNPR and/or } \\
\text { DNPatR }\end{array}$ & $\begin{array}{l}\text { Only DNPR } \\
\text { and/or } \\
\text { DNPatR }\end{array}$ & \multicolumn{2}{|l|}{ Total } \\
\hline \multirow[t]{2}{*}{ n (\%) } & n (\%) & n (\%) & n (\%) & \\
\hline & & & DCCG & $\begin{array}{l}+ \text { DNPR } \\
\text { and/or } \\
\text { DNPatR }\end{array}$ \\
\hline $213(45)$ & $153(33)$ & $102(22)$ & $366(78)$ & $102(22)$ \\
\hline
\end{tabular}

Notes: The Danish Colorectal Cancer Group (DCCG) Database, The Danish National Patient Registry (DNPR) and the Danish National Pathology Registry (DNPatR) During 2015-2015.

Table 3 The Completeness and Positive Predictive Value

\begin{tabular}{|l|l|l|l|l|l|}
\hline \multirow{2}{*}{ Registry } & \multicolumn{2}{|l|}{ DCCG } & \multirow{2}{*}{ Total } & Completeness & PPV \\
\cline { 2 - 3 } & $\mathbf{n = 3 6 6}$ & & & \\
\cline { 2 - 3 } & + & - & & & \\
\hline $\begin{array}{l}\text { DNPR and/or } \\
\text { DNPatR }\end{array}$ & 153 & 102 & 255 & $42(37-47)$ & $60(54-66)$ \\
DNPR & 118 & 89 & 207 & $\begin{array}{l}32(27-37) \\
19(15-23)\end{array}$ & $\begin{array}{l}57(50-64) \\
76(68-85)\end{array}$ \\
\hline DNPatR & 71 & 22 & 93 & 19 \\
\hline
\end{tabular}

Notes: The completeness: number of patients registered in the both the Danish Colorectal Cancer Group (DCCG) and each registry (the Danish National Patient Registry (DNPR) and the Danish National Pathology Registry (DNPatR)) (numerator), divided by the number $(n=366)$ registered in the DCCG (denominator). The positive predictive value (PPV) was calculated as the number of patients with a registered diagnosis in both the DCCG and each registry, divided by the total number of patients registered with a diagnosis of peritoneal metastases in the respective registry.

registered in the DCCG, the diagnosis of S-PM was registered concurrently; therefore, the median day was 0 (IQR: $0-0$ ). When the S-PM diagnosis was registered only in the DNPR/DNPatR, the median interval was 22 days (IQR: 7-116), while the median interval was 6 days (IQR: 0-19) when S-PM was registered in both the DCCG and the DNPR and/or the DNPatR (see Appendix for Illustration).

\section{Discussion}

In present study, we used the DCCG database as a reference to validate the registration of the ICD-10 codes and the SNOMED codes for PM in the DNPR/DNPatR. Using a combination of both the DNPR/DNPatR we found a completeness of $42 \%$ and a PPV of $60 \%$. In the DNPR/ DNPatR, some subgroups were found to be reported with a higher completeness; patients with age $<60$ years, WHO performance status 0 and no distant metastases. The DNPR and the DNPatR incompletely capture patients with S-PM.
Clinicians should be encouraged to register the presence of S-PM to increase the validity of register-based S-PM data.

We found that the completeness of S-PM registration is low in the DNPR and/or the DNPatR. However, advanced $\mathrm{CRC}$ and the registration of metastases at a specific anatomic location from any cancer disease are poorly investigated and even suggested to be underestimated in cancer registries. ${ }^{22}$ It has been investigated in an epidemiological study from 2009, which assessed the validity of the specific ICD-10 code for bone metastases originating from breast and prostate cancer. They found a sensitivity of the DNRP-registered ICD-10 code for bone metastases from prostate cancer to be $44 \%$ and $32 \%$ from breast cancer, revealing that it fails to capture more than half of the patients with bone-specific metastases. ${ }^{23}$ By the use of our algorithm, we found a similar tendency with a completeness of $32 \%$ when using only the DNPR, reaching only $42 \%$ when the DNPR was combined with the DNPatR.

Explanations for the underreporting of the registration of PM in the DNPR and the DNPatR might be several; when cancer patients present with metastases, these can be located at multiple sites, eg, liver and lungs and peritoneum, and thus with limited treatment options. In such cases, the clinicians' incentive to report all metastases is sparse, and often only those metastases that can be treated are reported to the national registries. ${ }^{24}$ Further, recording of metastases may not be prioritized for patients with a limited life expectancy. ${ }^{24}$ This is supported by the stratified analysis showing a higher completeness of S-PM registrations among potential candidates for curative treatment options as CRS and HIPEC; age <60 years, WHO performance status 0 , and no distant metastases. Contrary, a lower completeness was found among patients aged $>80$ years, WHO performance status $\geq 2$ and distant metastasis (liver and/or lung). This group of patients is according to national guidelines not eligible for CRS and HIPEC, which is a treatment offered to a selected group of patients with few, curable metastasis ( $\leq 3$ curable liver metastasis, $\leq 2$ curable lung metastasis), physiological age $<75$ years and WHO performance status $<2 .{ }^{25}$ However, the indications for CRS and HIPEC treatment are not fixed and keeps evolving. ${ }^{26}$ Therefore, clinicians should be encouraged to register the presence of PM to ensure valid register-based data on PM from CRC in various patients.

The coding applied in the Danish registries is only used for evident medical disease. The diagnosis of PM is in its nature challenging to verify for clinicians; it does not 
Table 4 Stratified Analysis

\begin{tabular}{|c|c|c|c|c|c|}
\hline \multirow[t]{2}{*}{ Variable } & DCCG & $\begin{array}{l}\text { DCCG and } \\
\text { DNPR and/or } \\
\text { DNPatR }\end{array}$ & $\begin{array}{l}\text { DNPR and/or } \\
\text { DNPatR }\end{array}$ & Total & \multirow{2}{*}{$\begin{array}{l}\text { Completeness in the } \\
\text { DNPR and/or } \\
\text { DNPatR } \\
\%(95 \% \text { Confidence } \\
\text { Interval) }\end{array}$} \\
\hline & $n=213$ & $n=153$ & $n=102$ & $n=468$ & \\
\hline \multicolumn{6}{|l|}{ Age Groups } \\
\hline$<60$ & $29(14)$ & $39(25)$ & $2 \mathrm{I}(2 \mathrm{I})$ & $89(19)$ & $57(46-69)$ \\
\hline $60-69$ & $58(27)$ & $39(25)$ & $2 I(2 I)$ & $118(25)$ & $40(30-50)$ \\
\hline $70-74$ & $37(17)$ & $27(18)$ & $17(17)$ & $81(17)$ & $42(30-54)$ \\
\hline $75-80$ & $36(17)$ & $23(15)$ & $26(25)$ & $85(18)$ & $39(27-5 I)$ \\
\hline$>80$ & $53(25)$ & $25(16)$ & $17(17)$ & $95(20)$ & $32(22-42)$ \\
\hline \multicolumn{6}{|l|}{ Sex } \\
\hline Female & $116(54)$ & $74(48)$ & $4 \mid(40)$ & $231(49)$ & $39(32-46)$ \\
\hline Male & $97(46)$ & $79(52)$ & $61(60)$ & $237(5 \mathrm{I})$ & $45(38-52)$ \\
\hline \multicolumn{6}{|c|}{ WHO Performance Status } \\
\hline Status 0 & $70(38)$ & $59(39)$ & $48(47)$ & 177 (38) & $46(37-54)$ \\
\hline Status I & $61(29)$ & $50(33)$ & $26(25)$ & 137 (29) & $45(36-54)$ \\
\hline Status 2 & $38(18)$ & $18(12)$ & $14(14)$ & $70(15)$ & $32(20-44)$ \\
\hline Status $>2$ & $22(10)$ & $13(8)$ & $6(6)$ & $41(9)$ & $37(2 \mid-53)$ \\
\hline Missing & $22(10)$ & $13(8)$ & $8(8)$ & $43(9)$ & $37(2 \mid-53)$ \\
\hline \multicolumn{6}{|c|}{ Tumour Location } \\
\hline Colon & $185(87)$ & $139(91)$ & $85(83)$ & $409(87)$ & $43(38-48)$ \\
\hline Rectum & $28(13)$ & $14(9)$ & $17(17)$ & $59(13)$ & $33(19-48)$ \\
\hline \multirow{2}{*}{\multicolumn{6}{|c|}{$\begin{array}{l}\text { Distant Metastases to Either } \\
\text { Lungs or Liver }\end{array}$}} \\
\hline & & & & & \\
\hline Yes & 143 (67) & $57(37)$ & $4 \mid(40)$ & $24 \mid(5 I)$ & $29(22-35)$ \\
\hline No & $70(33)$ & $96(63)$ & $61(60)$ & 227 (49) & $58(50-65)$ \\
\hline
\end{tabular}

Notes: Number of patients (\%) registered in I) Only the Danish Colorectal Cancer Group (DCCG), 2) Both the DCCG and the Danish National Patient Registry (DNPR) and/or Danish National Pathology Registry (DNPatR), 3) Only the DNPR and/or the DNPatR and 4) the total number of patients registered with synchronous peritoneal metastases. The Completeness of the registrations in the DNPR and/or the DNPatR: Number of patients registered in the both the DCCG and the DNPR and/or DNPatR (numerator), divided by the number registered in the DCCG (denominator).

necessarily rely on a histopathological verification, the sensitivity of a preoperative CT scan is low, and there is often a discrepancy between the radiological observed extent of peritoneal involvement and the findings during surgical exploration. ${ }^{11,27}$ Clinicians are only allowed to register diagnoses with a high degree of certainty, so a diagnosis that is rarely histopathological verified, as PM, may be underreported. Our results show that the completeness in the DNPatR is $19 \%$ but the PPV is $76 \%$. The low completeness in the DNPatR is potentially due to the fact that the majority of S-PM patients are not subjected to surgery, and therefore, a histopathological evaluation is lacking. Consequently, the majority of the registered S-PM diagnoses are most likely based on radiological findings or, in case of surgical exploration, the perioperative findings.
We developed a feasible algorithm to capture Danish patients with S-PM by combining the DCCG, the DNPR and the DNPatR. By combining the three registries, we found a 5\% prevalence of S-PM among CRC patients (Figure 1). This prevalence is in accordance with international literature, and demonstrates a similar and thereby representativeness of our algorithm. ${ }^{26}$ Yet, our results demonstrate that some subgroups are registered with a higher respective lower completeness in the DNPR/ DNPatR. Further, patients who were only registered in the DNPR/DNPatR differed with respect to age, sex, tumour and presence of distant metastases other than PM. Explanations for this variation might be several: First, clinicians are not obligated to mandatorily register the presence of PM in the Danish registries. In the DCCG, the registration is performed by dedicated surgeons with the purpose to ensure the quality 
of all types of treatment offered to Danish CRC patients. ${ }^{18}$ Contrary, the diagnoses in the DNPR/DNPatR are registered by any clinician respective pathologist during in-hospital treatment of the patient. Even though the registration process should be simple, there might be a different acceptability among surgeons, clinicians and pathologists. Second, we choose to distinguish between synchronous and metachronous peritoneal metastases 180 days after CRC diagnosis. Our results demonstrate a difference in the median time interval (and Interquartile range (IQR)) from CRC diagnosis to S-PM diagnosis according to each register. When registered only in the DNPR/DNPatR, the median of 22 days with a wide IQR (7-116) compared to a median of 6 days with a narrow IQR (0-19) when registered in both the DCCG and the DNPR/DNPatR. Causes to this difference are unknown and explanations are speculative. A histopathological examination takes, as a minimum, 3 days to conduct, which might explain the delay in S-PM registration. On the other hand, a median of 22 days with a wide IQR may indicate that the PM barely has been evident at the time of CRC diagnosis. It is plausible that the diagnosis of PM has not been detected during resection of the primary tumour, but diagnosed as an incidental finding by histopathology of the resected tumour. In such case, with a histopathological identified PM diagnosis, a detailed examination of the possibilities for postoperative treatment might explain the delay in the diagnosis of S-PM. Hypothetically, a postoperative treatment could include CRS and HIPEC performed approximately 3 months, as a minimum, after the primary resection of the $\mathrm{CRC}$ cancer. This demonstrates a difference in the timeliness (ie, how quickly data flow from "real-time" to the register) according to each register, and should be taken into consideration in future register-based studies of S-PM patients.

\section{Strengths and Limitations}

The study is based on a national cohort of CRC patients from the DCCG database known with a high completeness of CRC patients, demonstrating a high acceptability for surveillance of CRC patients in the DCCG. To our knowledge, this is the first both national and international study describing the quality of S-PM registration among CRC patients.

We excluded patients with non-CRC, thereby ensuring that the ICD-10 codes represented PM originated from CRC. Our algorithm combines 3 variables from the DCCG, 2 ICD10 codes and several SNOMED codes presented in the Appendix. The simplicity of the combination is high and should be reproducible for a potential external validation.
However, there is no perfect gold standard to identify S-PM, and consequently this limits the interpretation of the sensitivity of our algorithm applied. The value of medical charts as the golden standard has been questioned when searching for register-based metastases. ${ }^{15}$

We searched for patients with S-PM in a time period, where the awareness of PM and its treatment options have increased, ${ }^{28}$ and the DCCG database has undergone revisions. Our algorithm combines the Danish medical registries and shows flexibility in accordance with the expansion in knowledge, subsequently the changes in each registry. However, changes may introduce misclassification of S-PM during the early phase of an implementation. Hypothetically, $\mathrm{PM}$ is registered with a lower completeness in the DNPR/DNPatR in periods where the focus on PM has been minimal. This potential source of bias should be taken into consideration in future analytical studies investigating an association between S-PM and a specific research question. Further, our results from the stratified analysis show that the registration of S-PM differs within subgroups (eg, a higher completeness of S-PM registrations among patients being potentially eligible for curative treatment options as CRS and HIPEC), thereby introducing potential differential misclassification. This may also have implications for future studies investigating the prognosis of S-PM. The effect of the differential misclassification, ie, under-/or overestimation of an association, depends on whether S-PM is used as an exposure or outcome. For example, if a study investigates survival after CRC surgery and register-based S-PM data are used as an exposure variable (or disease status), the association between S-PM and survival will be underestimated because patients with a good prognosis are registered with a higher completeness compared to patients with a poor prognosis.

Further, we interpreted ovarian metastases as PM, which is a debated subject internationally. ${ }^{29}$ Finally, the current study only investigates the quality of peritoneal metastases registered synchronously with the CRC diagnosis; therefore, our results cannot be extrapolated to other populations, eg, register-based detection of recurrence in the peritoneum (metachronous peritoneal metastases).

\section{Conclusion}

The DNPR/DNPatR captures under half of patients with colorectal synchronous peritoneal metastases. Patients being potentially eligible for curative treatment options; patients with age $<60$ years, WHO performance status 0 
and no distant metastasis (other than PM) are registered with a higher completeness.

\section{Abbreviations}

CRC, colorectal cancer; PM, peritoneal metastases; S-PM, synchronous peritoneal metastases; DNPR, The Danish National Patient Registry; DCCG, The Danish Colorectal Cancer Group; DNPatR, The Danish National Pathology Registry.

\section{Acknowledgments}

The study was supported by the Program for Clinical Research Infrastructure (PROCRIN) established by the Lundbeck Foundation and the Novo Nordisk Foundation and administered by the Danish Regions.

\section{Author Contributions}

The corresponding author performed the data analysis. All authors contributed with a critical review of the data analysis and drafted/revised the article. All authors gave final approval of the version to be published and agreed to be accountable for all aspects of the work.

\section{Funding}

The "Manufacturer Einar Willumsen's Memorial Fund" financially supported the extraction of data. The funding agencies had no role in the design of the study; the collection, analysis, and interpretation of the data; the writing of the article; or the decision to submit the article for publication.

\section{Disclosure}

The authors declare no conflicts of interest in this work.

\section{References}

1. Weitz J, Koch M, Debus J, Hohler T, Galle PR, Buchler MW. Colorectal cancer. Lancet. 2005;365(9454):153-165. doi:10.1016/ S0140-6736(05)17706-X

2. Iversen LH. Aspects of survival from colorectal cancer in Denmark. Dan Med J. 2012;59(4):B4428.

3. Golfinopoulos V, Salanti G, Pavlidis N, Ioannidis JP. Survival and disease-progression benefits with treatment regimens for advanced colorectal cancer: a meta-analysis. Lancet Oncol. 2007;8 (10):898-911. doi:10.1016/S1470-2045(07)70281-4

4. Boysen AK, Spindler K-L, Hoyer M, et al. Metastasis directed therapy for liver and lung metastases from colorectal cancer - a population based study. Int J Cancer. 2018;143:3218-3226. doi:10.1002/ijc.31626

5. Iversen LH, Green A, Ingeholm P, Osterlind K, Gogenur I. Improved survival of colorectal cancer in Denmark during 2001-2012 - the efforts of several national initiatives. Acta Oncol. 2016;55(Suppl 2):10-23. doi:10.3109/0284186X.2015.1131331
6. Verwaal VJ, Bruin S, Boot H, van Slooten G, van Tinteren H. 8-year follow-up of randomized trial: cytoreduction and hyperthermic intraperitoneal chemotherapy versus systemic chemotherapy in patients with peritoneal carcinomatosis of colorectal cancer. Ann Surg Oncol. 2008;15(9):2426-2432. doi:10.1245/s10434-008-9966-2

7. Weber T, Roitman M, Link KH. Current status of cytoreductive surgery with hyperthermic intraperitoneal chemotherapy in patients with peritoneal carcinomatosis from colorectal cancer. Clin Colorectal Cancer. 2012;11(3):167-176. doi:10.1016/j.clcc.2012. 01.001

8. Neo EL, Beeke C, Price T, et al. South Australian clinical registry for metastatic colorectal cancer. ANZ J Surg. 2011;81(5):352-357. doi:10.1111/ans.2011.81.issue-5

9. Wang X, Hershman DL, Abrams JA, et al. Predictors of survival after hepatic resection among patients with colorectal liver metastasis. $\mathrm{Br}$ J Cancer. 2007;97(12):1606-1612. doi:10.1038/sj.bjc.6604093

10. Koumpa FS, Xylas D, Konopka M, et al. Colorectal peritoneal metastases: a systematic review of current and emerging trends in clinical and translational research. Gastroenterol Res Pract. 2019;2019:5180895. doi:10.1155/2019/5180895

11. Dresen RC, De Vuysere S, De Keyzer F, et al. Whole-body diffusion-weighted MRI for operability assessment in patients with colorectal cancer and peritoneal metastases. Cancer Imaging. 2019;19(1):1. doi:10.1186/s40644-018-0187-z

12. Schmidt M, Schmidt SA, Sandegaard JL, Ehrenstein V, Pedersen L, Sorensen HT. The Danish National Patient Registry: a review of content, data quality, and research potential. Clin Epidemiol. 2015;7:449-490. doi:10.2147/CLEP.S91125

13. Lash TL, Riis AH, Ostenfeld EB, Erichsen R, Vyberg M, Thorlaciusussing $\mathrm{O}$. A validated algorithm to ascertain colorectal cancer recurrence using registry resources in Denmark. Int J Cancer. 2015;136 (9):2210-2215. doi:10.1002/ijc.v136.9

14. Ording AG, Heide-jorgensen U, Christiansen CF, Norgaard M, Acquavella J, Sorensen HT. Site of metastasis and breast cancer mortality: a Danish nationwide registry-based cohort study. Clin Exp Metastasis. 2017;34(1):93-101. doi:10.1007/s10585-016-9824-8

15. Ehrenstein V, Hernandez RK, Maegbaek ML, et al. Validation of algorithms to detect distant metastases in men with prostate cancer using routine registry data in Denmark. Clin Epidemiol. 2015;7:259-265. doi:10.2147/CLEP.S74991

16. Vandenbroucke JP, von Elm E, Altman DG, et al. Strengthening the reporting of observational studies in epidemiology (STROBE): explanation and elaboration. Int $J$ Surg. 2014;12(12):1500-1524. doi:10.1016/j.ijsu.2014.07.014

17. Schmidt M, Schmidt SAJ, Adelborg K, et al. The Danish health care system and epidemiological research: from health care contacts to database records. Clin Epidemiol. 2019;11:563-591. doi:10.2147/ CLEP.S179083

18. Ingeholm P, Gogenur I, Iversen LH. Danish colorectal cancer group Database. Clin Epidemiol. 2016;8:465-468. doi:10.2147/CLEP.S99481

19. Schmidt M, Pedersen L, Sorensen HT. The Danish civil registration system as a tool in epidemiology. Eur J Epidemiol. 2014;29 (8):541-549. doi:10.1007/s10654-014-9930-3

20. Erichsen R, Lash TL, Hamilton-dutoit SJ, Bjerregaard B, Vyberg M, Pedersen L. Existing data sources for clinical epidemiology: the Danish National Pathology Registry and Data Bank. Clin Epidemiol. 2010;2:51-56. doi:10.2147/clep.S9908

21. Thygesen SK, Christiansen CF, Christensen S, Lash TL, Sorensen HT. The predictive value of ICD-10 diagnostic coding used to assess Charlson comorbidity index conditions in the population-based Danish National Registry of Patients. BMC Med Res Methodol. 2011;11:83. doi:10.1186/1471-2288-11-83

22. Oppelt KA, Luttmann S, Kraywinkel K, Haug U. Incidence of advanced colorectal cancer in Germany: comparing claims data and cancer registry data. BMC Med Res Methodol. 2019;19(1):142. doi:10.1186/s12874-019-0784-y 
23. Jensen AO, Norgaard M, Yong M, Fryzek JP, Sorensen HT. Validity of the recorded International Classification of Diseases, 10th edition diagnoses codes of bone metastases and skeletal-related events in breast and prostate cancer patients in the Danish National Registry of Patients. Clin Epidemiol. 2009;1:101-108. doi:10.2147/CLEP. S5446

24. Chawla N, Yabroff KR, Mariotto A, McNeel TS, Schrag D, Warren JL. Limited validity of diagnosis codes in Medicare claims for identifying cancer metastases and inferring stage. Ann Epidemiol. 2014;24(9):666672, 672 e661-662. doi:10.1016/j.annepidem.2014.06.099

25. (Danish) DCCG. National guidelines. Available from: https://dccg.dk/ wp-content/uploads/2018/12/2018-Peritoneal-karcinose-og-HIPEC -v-3_1.pdf. 2019 Accessed July 11, 2019.

26. Vassos N, Piso P. Metastatic colorectal cancer to the peritoneum: current treatment options. Curr Treat Options Oncol. 2018;19 (10):49. doi:10.1007/s11864-018-0563-8
27. Flicek K, Ashfaq A, Johnson CD, Menias C, Bagaria S, Wasif N. Correlation of radiologic with surgical peritoneal cancer index scores in patients with pseudomyxoma peritonei and peritoneal carcinomatosis: how well can we predict resectability?. J Gastrointest Surg. 2016;20(2):307-312. doi:10.1007/s11605-015-2880-6

28. Verwaal VJ, Rau B, Jamali F, et al. Registries on peritoneal surface malignancies throughout the world, their use and their options Int J Hyperthermia. 2017;33(5):528-533. doi:10.1080/02656736. 2017.1315178

29. Bignell MB, Mehta AM, Alves S, et al. Impact of ovarian metastases on survival in patients treated with cytoreductive surgery and hyperthermic intraperitoneal chemotherapy for peritoneal malignancy originating from appendiceal and colorectal cancer. Colorectal Dis. 2018;20(8):704-710. doi:10.1111/codi.2018.20.issue-8

\section{Publish your work in this journal}

Clinical Epidemiology is an international, peer-reviewed, open access, online journal focusing on disease and drug epidemiology, identification of risk factors and screening procedures to develop optimal preventative initiatives and programs. Specific topics include: diagnosis, prognosis, treatment, screening, prevention, risk factor modification,

Submit your manuscript here: https://www.dovepress.com/clinical-epidemiology-journal systematic reviews, risk \& safety of medical interventions, epidemiology \& biostatistical methods, and evaluation of guidelines, translational medicine, health policies \& economic evaluations. The manuscript management system is completely online and includes a very quick and fair peer-review system, which is all easy to use. 